\title{
Association Between Psychological Distress and Stress- Related Symptoms and Increased Risk of Type 2 Diabetes in Male Individuals: An Observational Study
}

\author{
Kaoru Takahashia, b, Tsutomu Kamino ${ }^{\mathrm{b}}$, Toshinari Yasuda ${ }^{\mathrm{b}, \mathrm{c}}$, \\ Akiko Suganuma $^{\mathrm{a}}$, Naoki Sakane ${ }^{\mathrm{a}, \mathrm{d}}$
}

\begin{abstract}
Background: This study evaluated the association between psychological distress and development of type 2 diabetes (T2D) among male individuals in the workplace.

Methods: This observational cohort study enrolled 6,326 male participants aged 18 - 65 years (mean age $47.4 \pm 9.5$ years, body mass index $23.4 \pm 3.4 \mathrm{~kg} / \mathrm{m}^{2}$, hemoglobin A1c $5.5 \pm 0.3 \%$ ), who received annual health checkups from April 2016 to March 2017. Those who had a hemoglobin A1c level $\geq 6.5 \%$, previous history of diabetes, or used diabetes medication were excluded from the analysis. Psychological distress was measured using the Brief Job Stress Questionnaire. Multivariate Cox proportional hazard regression models were used to estimate the development of T2D in relation to psychological distress and stress-related symptoms. The calculated hazard ratio (aHR) was adjusted for age, body mass index, and hemoglobin A1c level.
\end{abstract}

Results: During a mean follow-up period of 1.9 years, the incidence rate of new-onset T2D was $2.0 \%$. Baseline psychological parameters did not differ between participants with or without new-onset T2D. Depression was associated with an increased risk of T2D $(\mathrm{aHR}=$ $1.54,95 \%$ confidence interval $(\mathrm{CI}): 1.07-2.22)$, whereas vigor, irritation, fatigue, and anxiety were not. Moreover, inability to handle work $(\mathrm{aHR}=2.18,95 \% \mathrm{CI}$ : $1.14-4.19)$, sadness $(\mathrm{aHR}=1.93,95 \%$ CI: 1.14 - 3.26), headache (aHR $=1.98,95 \%$ CI: 1.18 - 3.34), shoulder stiffness $(\mathrm{aHR}=1.56,95 \% \mathrm{CI}: 1.10-2.23)$, and constipation/ diarrhea $(\mathrm{aHR}=1.71,95 \% \mathrm{CI}: 1.04-2.80)$ were associated with T2D incidence.

Manuscript submitted November 12, 2020, accepted December 4, 2020

Published online December 18, 2020

${ }^{a}$ Division of Preventive Medicine, Clinical Research Institute, National Hospital Organization Kyoto Medical Center, Kyoto, Japan

${ }^{b}$ Hyogo Health Service Association, Hyogo, Japan

'Hyogo College Of Medicine, Hyogo, Japan

${ }^{\mathrm{d} C}$ Corresponding Author: Naoki Sakane, Division of Preventive Medicine, Clinical Research Institute, National Hospital Organization Kyoto Medical Center, 1-1 Mukaihata-cho, Fukakusa, Fushimi-ku, Kyoto 612-8555, Japan. Email: nsakane@gf6.so-net.ne.jp

doi: https://doi.org/10.14740/jocmr4392
Conclusions: Depression and stress-related symptoms were associated with an increased risk of T2D. Industrial physicians and health care providers should evaluate these factors during health checkups to lower the T2D incidence in this population

Keywords: Diabetes; Depression; Stress; Observational study

\section{Introduction}

Environmental and lifestyle changes, as well as population aging, generally account for the rapid global increase in the prevalence and incidence of type 2 diabetes (T2D) in recent decades [1]. Apart from obesity and sedentary lifestyle, psychological stress represents an important risk factor for the accelerated incidence of T2D in high-risk populations [2-8]. The Japanese government launched a new occupational health policy called the Stress Check Program, which includes health checkups for psychological distress and stress-related symptoms $[9,10]$, the association between psychological distress using the Stress Check Program and increased risk of T2D. On the other hand, patients with T2D commonly experience stress-related symptoms such as irritation, fatigue, anxiety, and depression [11-15]. However, the association between stress-related symptoms and increased risk of T2D in the workplace is unknown. Therefore, this study aimed to evaluate the association between psychological distress and stress-related symptoms with the development of T2D in a cohort of male individuals.

\section{Materials and Methods}

\section{Study design}

This observational study recruited 6,326 individuals who participated in the Stress Check Program and received annual health checkups. According to the Japan Standard Industrial Classification by the Ministry of Internal Affairs and Communications (20 divisions), $38.1 \%$ of the participants were engaged in wholesale and rental trade, $29.9 \%$ in manufacturing, $12.5 \%$ in transport and postal services, $10.0 \%$ in government 
except elsewhere classified, and $9.5 \%$ in other divisions in the 99 collaborating companies and other industries. The inclusion criteria were male sex and age 18 - 65 years. Exclusion criteria were hemoglobin A1c (HbA1c) level $\geq 6.5 \%$, previous history of diabetes, and use of diabetes medication. We did not exclude subjects who had undergone psychotherapy (either counseling or medications) at baseline. Baseline surveys were conducted from April 2016 to March 2017. The annual health checkups and stress check surveys were conducted on different days. Self-reported stress-related symptoms considered symptoms presented within the past month.

\section{Outcome variables}

We evaluated the following anthropometric and biochemical variables: height, body weight, body mass index (BMI), blood pressure, HbA1c, high-density lipoprotein (HDL) cholesterol, and triglycerides. The self-administered questionnaire items on lifestyle behavior (smoking and exercise) were defined based on the definitions used for the Specific Health Checkup [16]. Diabetes was defined as a HbA1c level $\geq 6.5 \%$ or use of diabetes medication according to recent American Diabetes Association recommendations [17]. Questionnaire items on lifestyle habits were based on the standardized questionnaire used in the National Health Promotion Program [18, 19]. Psychological distress and stress-related symptoms were measured using the validated Brief Job Stress Questionnaire (BJSQ) [20], comprising 29 items assessing vigor (three items), anger-irritation (three items), fatigue (three items), anxiety (three items), depression (six items), and somatic symptoms (11 items). All items were measured on a 4-point Likert scale ranging from 1 (never) to 4 (almost always); points 1 and 2 received a score of 0 , and points 3 and 4 received a score of 1 . An individual was considered positive for depression when they scored at least 1 in each of the depression-related items. The BJSQ questionnaire items can measure six stress response scales; each item was developed based on previously standardized/authorized questionnaires. Specifically, "vigor," "fatigue," and "irritability" were based on the Profile of Mood States; "depression" was based on the Center for Epidemiologic Studies for Depression scale [21]; "anxiety" was based on the State-Trait Anxiety Inventory [22]; and "somatic stress responses" was based on the Screener for the Somatoform Disorders and the Subjective Well-being Inventory [23].

\section{Statistical analysis}

Data analysts were not blinded after the database was locked for final analysis. The Statistical Package for Social Science software version 24.0 (IBM SPSS Inc, Chicago, Illinois, USA) was used for all statistical analyses. Kaplan-Meier survival curves were compared by the generalized Wilcoxon test based on the depression status of the participants. The calculated hazard ratio (HR) was adjusted by age, BMI, and $\mathrm{HbAlc}(\mathrm{aHR})$, and is presented with its corresponding $95 \%$ confidence intervals (CI). Furthermore, we calculated the HR adjusted for confounding factors such as age, BMI, HbA1c, systolic blood pressure, tri- glycerides (TG) level, and exercise habits. Student's $t$-test (or the Mann-Whitney U test according to the frequency distribution of the variable) was used to compare the means (or distributions) of the two study arms for continuous variables. The $\chi^{2}$ test was used to compare the proportions of categorical variables. Relationships were analyzed using Spearman correlation coefficient. Missing values were deleted from the statistical analysis. A P value $<0.05$ was considered statistically significant.

This study protocol was approved by the Ethics Committee of Hyogo Yobouigaku association (approval number H283 ), and was conducted in compliance with the ethical standards of the responsible institution on human subjects as well as with the Helsinki Declaration.

\section{Results}

The baseline characteristics of the 6,326 participants included in the final analysis were as follows: mean age $47.4 \pm 9.5$ years, BMI $23.4 \pm 3.4 \mathrm{~kg} / \mathrm{m}^{2}$, and HbA1c $5.5 \pm 0.3 \%$. The average follow-up length was $1.9 \pm 0.4$ years, with a drop-out rate of $13.3 \%$ (Fig. 1). Over the study period, the incidence of new-onset T2D in the cohort participants was $2.0 \%$ (Table 1). Depression was associated with an increased risk of T2D $(\mathrm{aHR}=1.54 ; 95 \% \mathrm{CI}$ : 1.07 - 2.22), whereas vigor, irritation, fatigue, and anxiety were not associated with it (Table 2). In the depression assessment scale, scoring points on the items "I have been unable to handle work" $(\mathrm{aHR}=2.18$; 95\% CI: 1.14 - 4.19) and "I have felt sad" $($ aHR $=1.93 ; 95 \%$ CI: 1.14 - 3.26) were associated with an increased risk of T2D (Table 3). Regarding stress-related symptoms, headache $(\mathrm{aHR}=1.98 ; 95 \% \mathrm{CI}: 1.18$ - 3.34), shoulder stiffness $(\mathrm{aHR}=1.56 ; 95 \% \mathrm{CI}: 1.10-2.23)$, and constipation/ diarrhea $(\mathrm{aHR}=1.71 ; 95 \% \mathrm{CI}: 1.04-2.80)$ were associated with an increased risk of T2D, whereas other symptoms did not show any association with it (Table 2). Depression was not associated with an increased risk of T2D in participants with fasting plasma glucose $(\mathrm{FPG}) \geq 110 \mathrm{mg} / \mathrm{dL}(\mathrm{aHR}=1.71,95 \% \mathrm{CI}: 0.82-3.57)$ or $\mathrm{FPG}$ of $<110 \mathrm{mg} / \mathrm{dL}(\mathrm{aHR}=1.33,95 \% \mathrm{CI}$ : $0.49-3.55)$. The cumulative incidence of T2D was significantly higher in participants with depression than in those without depression $(\mathrm{P}=0.037$, Fig. 2$)$. The cumulative incidence of T2D was significantly higher in participants with depression than in those without depression with $\mathrm{HbA} 1 \mathrm{c} \geq 5.7 \%(\mathrm{P}=0.010)$. However, the incidence was higher but not significant in participants with depression than in those without depression with $\mathrm{HbA} 1 \mathrm{c}<5.7 \%$ $(\mathrm{P}=0.096)$. Depression was not associated with an increased risk of T2D $(\mathrm{aHR}=1.50,95 \% \mathrm{CI}: 0.93-2.41)$ after adjusting for the confounding factors. Skipping breakfast correlated with the anger-irritability, fatigue, anxiety, and depression scores, but not with vigor. Late-night dinner eating also correlated with the anger-irritability, fatigue, anxiety, and depression scores, but not with vigor. Alcohol consumption habit did not correlate with psychological distress and depression (Table 4).

\section{Discussion}

This study demonstrated the association of depression symp- 


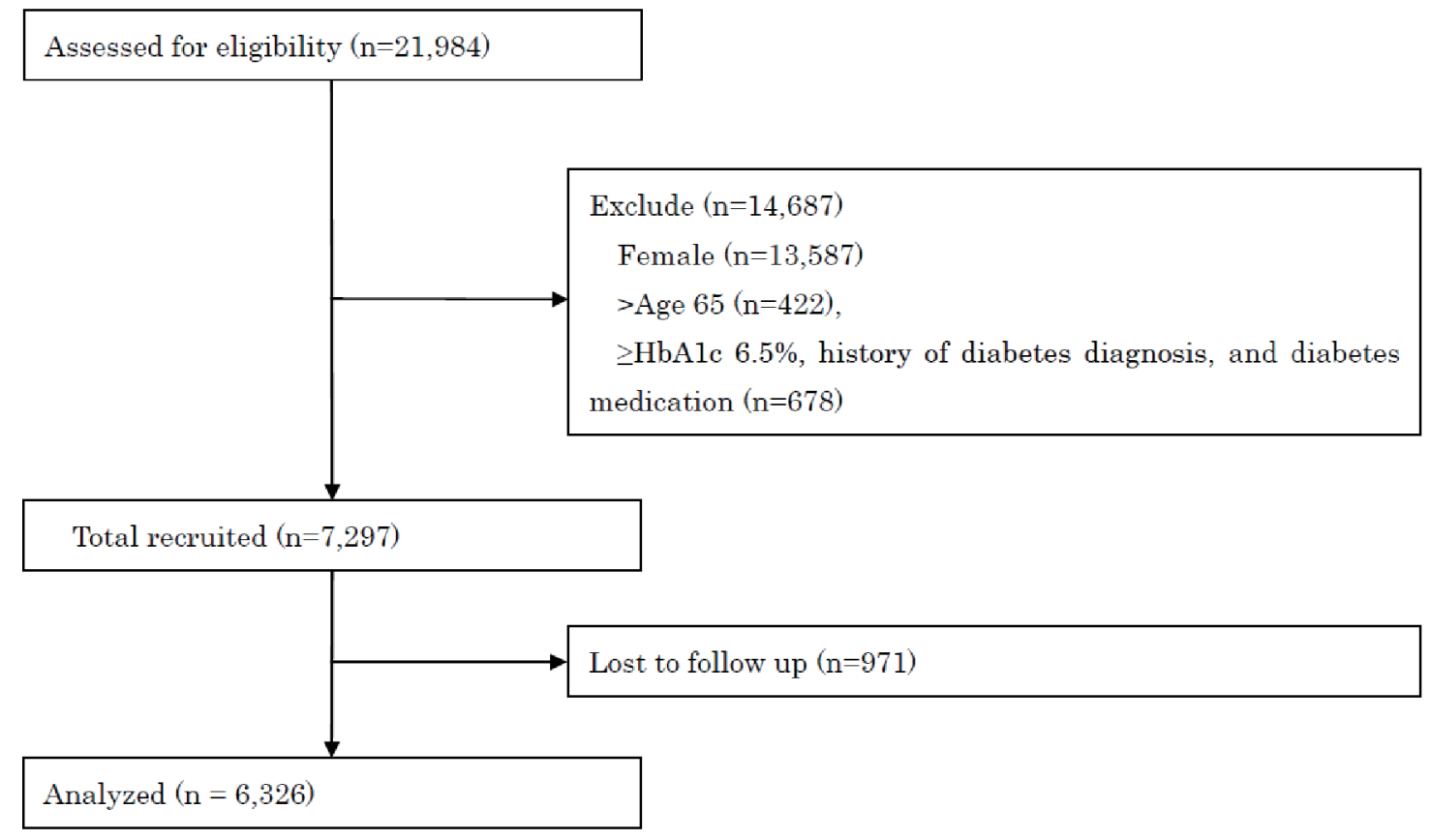

Figure 1. STROBE flow-chart of participants. STROBE: Strengthening the Reporting of Observational Studies in Epidemiology; HbA1c: HbA1c: hemoglobin A1c.

toms, especially sadness and inability to handle work, with the development of diabetes. Increasing evidence indicates that depression and T2D share biological origins, particularly overactivation of innate immunity leading to a cytokine-mediated inflammatory response, and potential dysregulation of the hypothalamic-pituitary-adrenal axis [24]. Over time, these pathways can lead to insulin resistance, cardiovascular disease, depression, and increased risk of T2D [25]. Therefore, our results are consistent with previous studies.

In this study, we also found that sadness was associated with an increased risk of T2D. Although sadness is a normal emotional expression, it can be an indication of serious medical conditions that is easily missed by most clinicians [26]. Sadness was often identified as part of depression scales and as a predictor of cardiac events [27]. However, the mechanisms through which sadness affects the development of T2D are unknown. Sources of sadness are complex and multifactorial (e.g., biological, psychosocial, and environmental). Moreover, sadness and expressions of sadness have been linked to neuroanatomical and neurophysiologic functions, and have been associated with increased activity in the left amygdala and right temporal lobe [28]. Older adults often deny feeling sad while exhibiting other characteristics of depression [29]. Proinflammatory cytokines such as interleukin (IL)-1 are known to generate sadness, while daily happiness significantly predicted lower baseline and post-stress IL-6 after controlling for daily sadness in T2D [30,31]. Low-grade inflammation was a significant cause of insulin resistance and beta cell failure [32]. Therefore, sadness may play an important role in the development of diabetes in the age group included in this study (18 - 65 years). Further studies including large samples are required to elucidate the association between sadness and the development of T2D.

Another depression-related factor, inability to handle work, was associated with an increased risk of T2D. Long working hours were associated with a higher risk of T2D [33]. The job demands-control model was associated with higher levels of blood pressure and serum lipids among the Japanese working population [34], but job control was not associated with a higher risk of developing T2D [35]. The reason behind the discrepancy between the results of this study and of previous studies is unknown. We hypothesize that differences in work conditions may partly explain the distinct results.

Stress-related symptoms, such as headache and shoulder stiffness, were also associated with T2D development. The relationship between headaches and the development of T2D is unknown. Patients with T2D experience changes in vascular reactivity and nerve conduction that may be relevant for migraine pathophysiology. These changes may also occur in individuals with prediabetes. However, previous studies on the relationship between headaches and T2D are contradictory [36]. Further studies employing a headache scale [37] are necessary to clarify this association. Furthermore, the prevalence of shoulder stiffness is increased in people with T2D [38].

Finally, we observed that bowel dysfunction symptoms, including diarrhea and constipation, were associated with an increased risk of developing T2D. Diabetes is associated with constipation, and poor glycemic control, diabetes duration, and BMI affect the risk of bowel dysfunction symptoms [39]. Although the exact mechanism of diabetic bowel dysfunction is unknown, autonomic nervous system dysfunction caused by hyperglycemia is hypothesized to influence colon motility.

We were unaware of the plausible reasons for job-related 
Table 1. Baseline Characteristics of the Study Participants

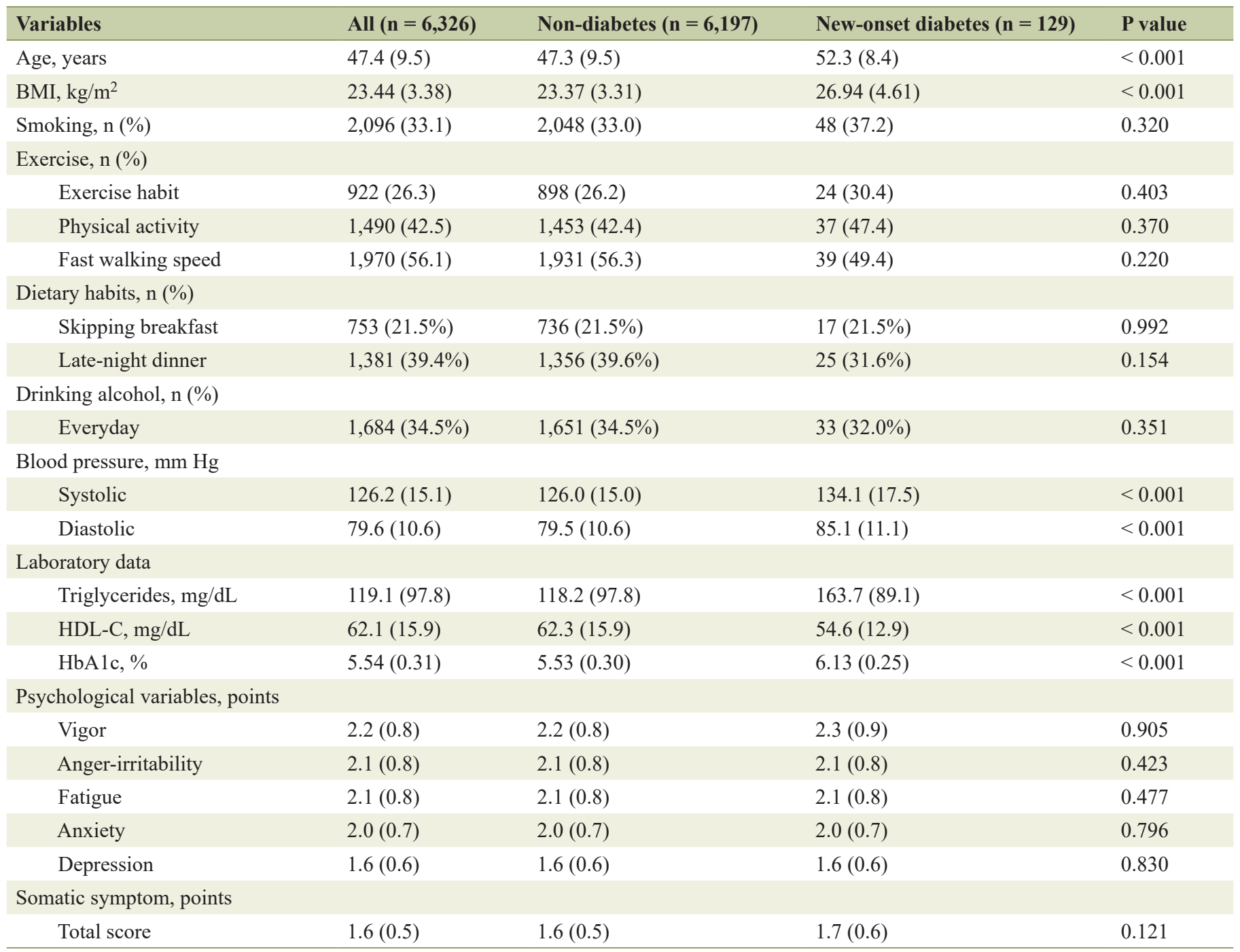

Mean (standard deviation) or n (\%). BMI: body mass index; HDL-C: high-density lipoprotein cholesterol; HbA1c: hemoglobin A1c.

stress. Several job-stress models such as job-demand-control, effort-reward-imbalance, and organizational justice have been proposed [40]. Further examination including job-stress models is required to confirm these issues in the future.

In this study, skipping breakfast and late-night dinner eating correlated with depression scores. Skipping breakfast was associated with depression among adults with different socioeconomic factors [41]. Although the exact mechanism by which breakfast contributes to reducing depression and stress remains unclear, several mechanisms have been proposed. After breakfast, carbohydrates are converted into glucose, producing changes in the levels of acetylcholine, serotonin, tryptophan, and cortisol. Ingestion of carbohydrates is beneficial for the brain after night fasting as it reduces cortisol production, thereby decreasing the "stress" signal [42]. Overeating at dinner has been correlated to stress responses (e.g., fatigue, anxiety, and depression) in Japanese male workers [43]. Further examination including dietary interventions such as eat- ing breakfast and reducing overeating at dinner are required to confirm these issues.

\section{Strengths and limitations}

The longitudinal design and use of a validated scale for measuring depression and stress status were the main strengths of this study. Conversely, the main limitations were as follows: 1) The cohort used in this study originated from the Hyogo Prefecture in Japan, which may affect the generalizability of our results, especially for populations outside of Japan; 2) The measured outcomes were self-reported, which may incur some inaccuracy; 3) The follow-up period was short; 4) Occupational information, such as shift-worker, desk work, etc., was unknown; 5) Although a meta-analysis indicated an association between antidepressant use and incidence of diabetes [44], we did not collect antidepressant usage information in 
Table 2. Adjusted Hazard Ratio for Developing Type 2 Diabetes

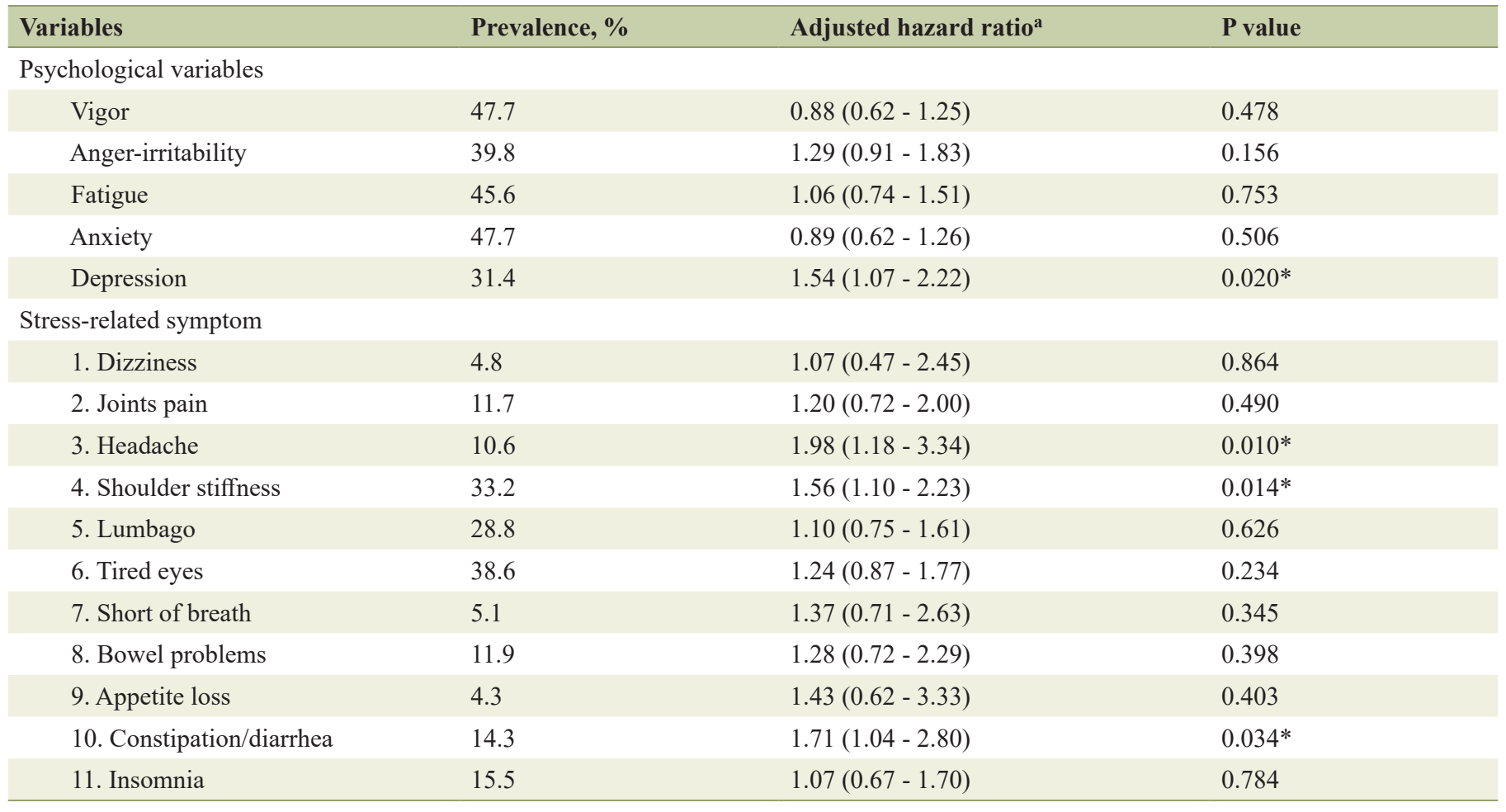

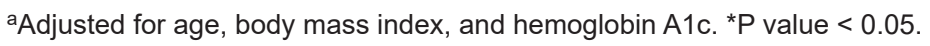

this study. To perform subgroup analysis, the sample size was small. These limitations should be considered when interpreting the results.

In conclusion, the findings of the current study indicated that depression and stress-related symptoms may increase the risk of developing T2D. Industrial physicians and health care providers should observe these factors during health checkups to potentially lower the incidence of T2D in this population.

\section{Acknowledgments}

We thank all the study investigators and staff and participants who participated in this study, for helpful discussions during manuscript development.

\section{Financial Disclosure}

This work was partly supported by JSPS KAKENHI (grant number: 18K01988).

\section{Conflict of Interest}

The authors have no conflicts of interest.

\section{Informed Consent}

All participants received a full explanation of the study proto-

Table 3. Adjusted Hazard Ratio for Developing Type 2 Diabetes According to the Depression Subscale

\begin{tabular}{|c|c|c|c|}
\hline Variables & Prevalence, $\%$ & Adjusted hazard ratio ${ }^{a}$ & P value \\
\hline \multicolumn{4}{|l|}{ Depression subscale } \\
\hline 1. I have been depressed & 19.8 & $1.18(0.77-1.82)$ & 0.441 \\
\hline 3. I have been unable to concentrate & 11.2 & $1.34(0.75-2.39)$ & 0.323 \\
\hline 4. I have felt gloomy & 20.5 & $1.30(0.83-2.02)$ & 0.252 \\
\hline 6. I have felt sad & 8.5 & $1.93(1.14-3.26)$ & $0.015^{*}$ \\
\hline
\end{tabular}

${ }^{a}$ Adjusted for age, body mass index, and hemoglobin $\mathrm{A} 1 \mathrm{c}$. ${ }^{*} \mathrm{P}$ value $<0.05$. 


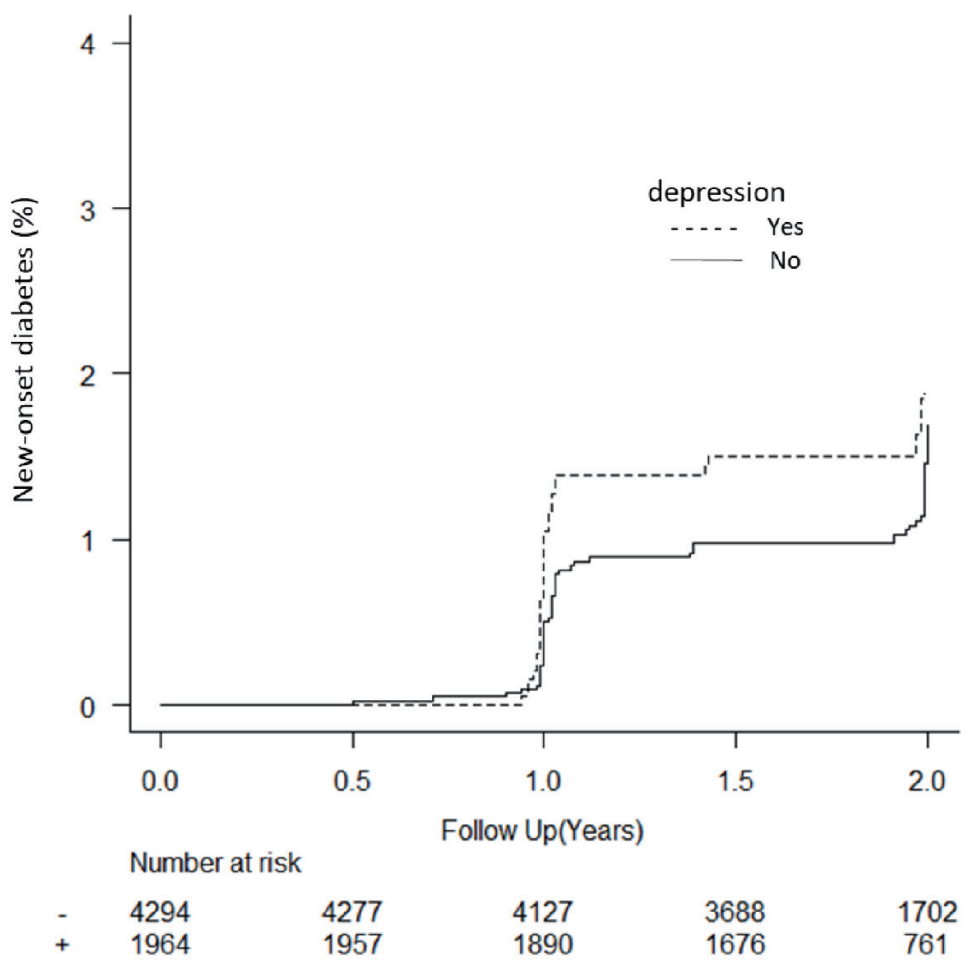

Figure 2. Kaplan Meier plot of new-onset diabetes in participants based on depression status. Sixty-eight participants without depression at baseline were excluded from the analysis.

Table 4. Correlation of Psychological Variables With Dietary Habits and Alcohol Consumption

\begin{tabular}{|c|c|c|c|c|c|}
\hline Variables & Vigor & Anger-irritability & Fatigue & Anxiety & Depression \\
\hline \multicolumn{6}{|l|}{ Dietary habits } \\
\hline Skipping breakfast & -0.001 & 0.065 & 0.059 & 0.055 & 0.076 \\
\hline Late-night dinner & -0.026 & 0.118 & 0.165 & 0.122 & 0.126 \\
\hline$P$ value & 0.133 & $<0.001$ & $<0.001$ & $<0.001$ & $<0.001$ \\
\hline$P$ value & 0.929 & 0.197 & 0.097 & 0.461 & 0.710 \\
\hline
\end{tabular}

col and provided informed consent.

\section{Author Contributions}

KT and NS conceived the ideas. KT analyzed the data. NS acquired the funding. KT, TK, and TY collected the data. NS and AS wrote the original draft. KT, TK, and TY reviewed and wrote the article.

\section{Data Availability}

The authors declare that data supporting the findings of this study are available within the article.

\section{Abbreviations}

BMI: body mass index; FPG: fasting plasma glucose; HDL-C: high-density lipoprotein cholesterol; T2D: type 2 diabetes

\section{References}

1. Kolb H, Martin S. Environmental/lifestyle factors in the pathogenesis and prevention of type 2 diabetes. BMC Med. 2017;15(1):131. 
2. Virtanen M, Ferrie JE, Tabak AG, Akbaraly TN, Vahtera J, Singh-Manoux A, Kivimaki M. Psychological distress and incidence of type 2 diabetes in high-risk and lowrisk populations: the Whitehall II Cohort Study. Diabetes Care. 2014;37(8):2091-2097.

3. Novak M, Bjorck L, Giang KW, Heden-Stahl C, Wilhelmsen L, Rosengren A. Perceived stress and incidence of Type 2 diabetes: a 35-year follow-up study of middleaged Swedish men. Diabet Med. 2013;30(1):e8-16.

4. Toshihiro M, Saito K, Takikawa S, Takebe N, Onoda T, Satoh J. Psychosocial factors are independent risk factors for the development of Type 2 diabetes in Japanese workers with impaired fasting glucose and/or impaired glucose tolerance. Diabet Med. 2008;25(10):1211-1217.

5. Heianza Y, Arase Y, Kodama S, Tsuji H, Fujihara K, Saito $\mathrm{K}$, Hara S, et al. Simple self-reported behavioral or psychological characteristics as risk factors for future type 2 diabetes in Japanese individuals: Toranomon Hospital Health Management Center Study 14. J Diabetes Investig. 2015;6(2):236-241.

6. Cosgrove MP, Sargeant LA, Caleyachetty R, Griffin SJ. Work-related stress and Type 2 diabetes: systematic review and meta-analysis. Occup Med (Lond). 2012;62(3):167173.

7. Sui H, Sun N, Zhan L, Lu X, Chen T, Mao X. Association between Work-Related Stress and Risk for Type 2 Diabetes: A Systematic Review and Meta-Analysis of Prospective Cohort Studies. PLoS One. 2016;11(8):e0159978.

8. Deleskog A, Ljung R, Forsell Y, Nevriana A, Almas A, Moller J. Severity of depression, anxious distress and the risk of type 2 diabetes - a population-based cohort study in Sweden. BMC Public Health. 2019;19(1):1174.

9. Kawakami N, Tsutsumi A. The Stress Check Program: a new national policy for monitoring and screening psychosocial stress in the workplace in Japan. J Occup Health. 2016;58(1):1-6.

10. Imamura K, Asai Y, Watanabe K, Tsutsumi A, Shimazu A, Inoue A, Hiro $\mathrm{H}$, et al. Effect of the National Stress Check Program on mental health among workers in Japan: A 1-year retrospective cohort study. J Occup Health. 2018;60(4):298-306.

11. Plummer MP, Finnis ME, Phillips LK, Kar P, Bihari S, Biradar V, Moodie S, et al. Stress induced hyperglycemia and the subsequent risk of type 2 diabetes in survivors of critical illness. PLoS One. 2016;11(11):e0165923.

12. Pashaki MS, Mezel JA, Mokhtari Z, Gheshlagh RG, Hesabi PS, Nematifard T, Khaki S. The prevalence of comorbid depression in patients with diabetes: A metaanalysis of observational studies. Diabetes Metab Syndr. 2019;13(6):3113-3119.

13. Reaney M, Elash CA, Litcher-Kelly L. Patient Reported Outcomes (PROs) used in recent Phase 3 trials for Type 2 Diabetes: A review of concepts assessed by these PROs and factors to consider when choosing a PRO for future trials. Diabetes Res Clin Pract. 2016;116:54-67.

14. Singh R, Teel C, Sabus C, McGinnis P, Kluding P. Fatigue in type 2 diabetes: impact on quality of life and predictors. PLoS One. 2016;11(11):e0165652.

15. Drivsholm T, de Fine Olivarius N, Nielsen AB, Siersma
V. Symptoms, signs and complications in newly diagnosed type 2 diabetic patients, and their relationship to glycaemia, blood pressure and weight. Diabetologia. 2005;48(2):210-214.

16. Fukasawa T, Tanemura N, Kimura S, Urushihara H. Utility of a specific health checkup database containing lifestyle behaviors and lifestyle diseases for employee health insurance in Japan. J Epidemiol. 2020;30(2):57-66.

17. American Diabetes Association. 2. Classification and diagnosis of diabetes: standards of medical care in diabetes-2020. Diabetes Care. 2020;43(Suppl 1):S14-S31.

18. Nakajima K, Suwa K. Association of hyperglycemia in a general Japanese population with late-night-dinner eating alone, but not breakfast skipping alone. J Diabetes Metab Disord. 2015;14:16.

19. Yoshimoto T, Ochiai H, Shirasawa T, Nagahama S, Uehara A, Muramatsu J, Kokaze A. Clustering of lifestyle factors and its association with low back pain: a crosssectional study of over 400,000 Japanese adults. J Pain Res. 2020;13:1411-1419.

20. Inoue A, Kawakami N, Shimomitsu T, Tsutsumi A, Haratani T, Yoshikawa T, Shimazu A, et al. Development of a short version of the new brief job stress questionnaire. Ind Health. 2014;52(6):535-540.

21. Radloff LS. The CES-D scale: a self-report depression scale for research in the general population. Appl Psych Meas. 1977;1:385-401.

22. Spielberger CD. Manual for the state-trait anxiety inventory STAI (Form Y) ("Self-Evaluation Questionnaire"). San Diego: Mindgarden. 1983.

23. Tonan K, Sonoda A, Ono Y. Production of the subjective well-being inventory Japanese Edition: it's reliability and validity. The Japanese Journal of Health Psychology. 1995;8:12-19. (in Japanese).

24. Moulton CD, Pickup JC, Ismail K. The link between depression and diabetes: the search for shared mechanisms. Lancet Diabetes Endocrinol. 2015;3(6):461-471.

25. Ho N, Sommers MS, Lucki I. Effects of diabetes on hippocampal neurogenesis: links to cognition and depression. Neurosci Biobehav Rev. 2013;37(8):1346-1362.

26. Lokko HN, Stern TA. Sadness: diagnosis, evaluation, and treatment. Prim Care Companion CNS Disord. 2014;16(6).

27. Doyle F, Conroy R, McGee H, Delaney M. Depressive symptoms in persons with acute coronary syndrome: specific symptom scales and prognosis. J Psychosom Res. 2010;68(2):121-130.

28. Blair RJ, Morris JS, Frith CD, Perrett DI, Dolan RJ. Dissociable neural responses to facial expressions of sadness and anger. Brain. 1999;122(Pt 5):883-893.

29. Gallo JJ, Rabins PV. Depression without sadness: alternative presentations of depression in late life. Am Fam Physician. 1999;60(3):820-826.

30. Francoeur RB. Symptom profiles of subsyndromal depression in disease clusters of diabetes, excess weight, and progressive cerebrovascular conditions: a promising new type of finding from a reliable innovation to estimate exhaustively specified multiple indicators-multiple causes (MIMIC) models. Diabetes Metab Syndr Obes. 2016;9:391-416. 
31. Panagi L, Poole L, Hackett RA, Steptoe A. Happiness and inflammatory responses to acute stress in people with type 2 diabetes. Ann Behav Med. 2019;53(4):309-320.

32. Kataria Y, Ellervik C, Mandrup-Poulsen T. Treatment of type 2 diabetes by targeting interleukin-1: a meta-analysis of 2921 patients. Semin Immunopathol. 2019;41(4):413425.

33. Kuwahara K, Imai T, Miyamoto T, Kochi T, Eguchi M, Nishihara A, Nakagawa T, et al. Sleep duration modifies the association of overtime work with risk of developing type 2 diabetes: Japan epidemiology collaboration on occupational health study. J Epidemiol. 2018;28(7):336340.

34. Kawakami N, Haratani T. Epidemiology of job stress and health in Japan: review of current evidence and future direction. Ind Health. 1999;37(2):174-186.

35. de Souza Santos R, Harter Griep R, Mendes da Fonseca MJ, Chor D, Santos IS, Melo ECP. Combined use of job stress models and the incidence of glycemic alterations (Prediabetes and Diabetes): results from ELSA-Brasil study. Int J Environ Res Public Health. 2020;17:1539.

36. Aamodt AH, Stovner LJ, Midthjell K, Hagen K, Zwart JA. Headache prevalence related to diabetes mellitus. The Head-HUNT study. Eur J Neurol. 2007;14(7):738-744.

37. Hunter M. The Headache Scale: a new approach to the assessment of headache pain based on pain descriptions. Pain. 1983;16(4):361-373.

38. Cole A, Gill TK, Shanahan EM, Phillips P, Taylor AW, Hill CL. Is diabetes associated with shoulder pain or stiff- ness? Results from a population based study. J Rheumatol. 2009;36(2):371-377.

39. Ihana-Sugiyama $\mathrm{N}$, Nagata $\mathrm{N}$, Yamamoto-Honda $\mathrm{R}$, Izawa E, Kajio H, Shimbo T, Kakei M, et al. Constipation, hard stools, fecal urgency, and incomplete evacuation, but not diarrhea is associated with diabetes and its related factors. World J Gastroenterol. 2016;22(11):32523260 .

40. Jarczok MN, Jarczok M, Mauss D, Koenig J, Li J, Herr RM, Thayer JF. Autonomic nervous system activity and workplace stressors - a systematic review. Neurosci Biobehav Rev. 2013;37(8):1810-1823.

41. Lee SA, Park EC, Ju YJ, Lee TH, Han E, Kim TH. Breakfast consumption and depressive mood: A focus on socioeconomic status. Appetite. 2017;114:313-319.

42. Ferrer-Cascales R, Sanchez-SanSegundo M, Ruiz-Robledillo N, Albaladejo-Blazquez N, Laguna-Perez A, Zaragoza-Marti A. Eat or Skip Breakfast? The important role of breakfast quality for health-related quality of life, stress and depression in Spanish adolescents. Int J Environ Res Public Health. 2018;15(8).

43. Suzuki A, Sakurazawa H, Fujita T, Akamatsu R. Overeating at dinner time among Japanese workers: Is overeating related to stress response and late dinner times? Appetite. 2016;101:8-14.

44. Salvi V, Grua I, Cerveri G, Mencacci C, Barone-Adesi F. The risk of new-onset diabetes in antidepressant users - A systematic review and meta-analysis. PLoS One. 2017;12(7):e0182088. 